\title{
The Role of Individual and Neighborhood Social Factors on Periodontitis: The Third National Health and Nutrition Examination Survey
}

\author{
Luisa N. Borrell, ${ }^{*}$ Brian A. Burt, ${ }^{\ddagger}$ Rueben C. Warren, $\S$ and Harold W. Neighbors $\|$
}

Background: This study investigates whether race/ethnicity, individual income, education, and neighborhood socioeconomic characteristics are independently associated with periodontitis in adults $\geq 18$ years of age who participated in the third National Health and Nutrition Examination Survey (NHANES III).

Methods: Analyses were limited to participants in NHANES III who self-identified as non-Hispanic black, non-Hispanic white, or Mexican American, received a periodontal examination, and whose records were linked to the 1990 U.S. Census data $(N=13,090)$. Periodontitis was investigated using a combination of clinical attachment loss and probing depth. Marginal logistic regression models were used to assess the association of race/ethnicity, individual income, education, and neighborhood socioeconomic characteristics with periodontitis before and after adjusting for selected covariates. A survey program was used to account for the survey sampling design and for the intraneighborhood correlation of outcomes of participants selected from the same neighborhood.

Results: Race/ethnicity, education, and neighborhood socioeconomic conditions were associated with periodontitis before and after controlling for selected covariates. After adjustment, blacks were twice (1.58 to 2.53 ) as likely to have periodontitis as whites. Compared to those with more than a high school education, those with less than a high school diploma were twice (1.48 to 2.89 ) as likely to have periodontitis. Individuals living in a neighborhood in the lowest tertile of the socioeconomic score were 1.81 times (1.36 to 2.41 ) more likely to have periodontitis than those living in a neighborhood in the highest tertile of the socioeconomic score.

Conclusion: This study indicates that race/ethnicity, individual education, and neighborhood socioeconomic circumstances are important for periodontal health. $J$ Periodontol 2006; 77:444-453.

\section{KEY WORDS}

Neighborhoods; periodontal diseases; periodontitis; socioeconomic status.

\footnotetext{
* Department of Epidemiology, Mailman School of Public Health, Columbia University, New York, NY.

† School of Dental and Oral Surgery, Columbia University.

\# Department of Epidemiology, School of Public Health, University of Michigan, Ann Arbor, MI.

$\S$ Interdenominational Theological Center, Atlanta, GA.

II Department of Health Behavior and Health Education, School of Public Health, University of Michigan.
}

$\mathrm{R}$ acial/ethnic and socioeconomic differences in periodontal diseases have been documented in the U.S. for years. ${ }^{1-12}$ African Americans exhibit worse periodontal conditions than their Hispanic and non-Hispanic white counterparts. Hispanics have similar prevalences of periodontal diseases to non-Hispanic whites. ${ }^{1,2,5,6,8,13-16}$ People with lower socioeconomic status (SES), regardless of their race/ethnicity, are more likely to have periodontal diseases than their higher SES peers. ${ }^{3-5,7,8,10,17}$

Several studies have documented an association between area-based socioeconomic indicators and health outcomes. ${ }^{18-20}$ Although the mechanisms by which area-based SES affects health are not well understood, it has been suggested that neighborhood socioeconomic conditions could influence the health behaviors and health-related beliefs of their residents independent of their personal SES. ${ }^{19,21}$ In the case of periodontal diseases, for example, neighborhood conditions could influence risky behaviors, such as smoking, and normative attitudes toward oral health, such as regular dental checkups. In addition, the SES level of a geographic area can influence the supply of health professionals and thereby increase or decrease access to care. ${ }^{22}$ However, existing data show that dental personnel and access to health are not correlated with better health

doi: 10.1902/jop.2006.050158 
status. ${ }^{23}$ No studies were found that investigated the role of race/ethnicity, socioeconomic indicators, and neighborhood socioeconomic conditions simultaneously on periodontal diseases at the national level.

The third National Health and Nutrition Examination Survey (NHANES III) affords the opportunity to investigate whether race/ethnicity, individual income, education, and neighborhood socioeconomic characteristics are independently associated with periodontal diseases before and after controlling for selected individual characteristics. Specifically, this article examines whether race/ethnicity, family income, individual education, and neighborhood socioeconomic conditions are independently associated with periodontitis before and after adjusting for selected individual characteristics and risk factors. In addition, we also examined whether risk factors for periodontal diseases, such as smoking and diabetes, explain the socioeconomic and racial/ethnic differences in periodontitis after adjusting for neighborhood SES circumstances. We expected race/ethnicity, income, education, and neighborhood SES conditions to be independently associated with periodontal diseases. In addition, we expected that the association of race/ ethnicity, education, and income with periodontal diseases depended on neighborhood SES conditions.

\section{MATERIALS AND METHODS}

Individual data for this study came from NHANES III (Adult and Examination files) public-use data files, and the neighborhood data were obtained from the 1990 U.S. Census. This survey assessed the health status of a nationally representative sample of the civilian non-institutionalized U.S. population, selected through a stratified multistage probability sampling design. Full descriptions of the sample design in NHANES III have been reported elsewhere. ${ }^{24}$ NHANES III yielded a sample of 20,050 persons $\geq 17$ years of age, of whom 16,833 were $\geq 18$ years of age and had some part of a dental examination. However, 1,342 subjects were excluded for medical reasons and an additional 1,826 individuals did not have information on their periodontal status. These exclusion criteria yielded a final sample of 13,665 records that were linked to census tract data. Once records were linked, this analysis was limited to the records of adults $\geq 18$ years who identified themselves as non-Hispanic black $(\mathrm{N}=3,986)$, non-Hispanic white $(\mathrm{N}=4,964)$, or Mexican American $(\mathrm{N}=4,140)$ during NHANES III. A final sample of 13,090 subjects in 1,126 census tracts (median: six participants per census tract; range: 1 to 147) was available for analyses.

For the dental examination, dentists trained in the survey examination protocol conducted the periodontal examinations. ${ }^{24}$ Briefly, the periodontal examination was conducted in two sites per tooth, mid-buccal and mesio-buccal, in two randomly chosen quad- rants, one maxillary and one mandibular, on the assumption that conditions in these two quadrants would represent the mouth. Third molars were excluded because of their frequent extraction in young adulthood, so a maximum of 14 teeth and 28 sites per individual were examined. Previous studies have used several combinations of clinical attachment loss (CAL) and probing depth (PD) to establish periodontitis case definitions. ${ }^{9,25,26}$ For this study, the distribution of CAL and PD was evaluated in the total population and in each racial/ethnic group in the survey. Prior to any hypotheses testing, we tested several case definitions before arriving at the one used in this analysis. A periodontitis case was defined as a person who had at least two sites with CAL $\geq 4 \mathrm{~mm}$ and at least one site with PD $\geq 4 \mathrm{~mm}$. However, these conditions did not have to be present in the same site or tooth.

The main independent variables of interest at the individual level were race/ethnicity, education, and income. Race/ethnicity was defined as non-Hispanic black, non-Hispanic white, and Mexican American. Hereafter, we will refer to non-Hispanic black and non-Hispanic white as black and white, respectively. Education was recorded as a continuous variable from 0 to 17 years of education and was categorized as $<12,12$, and $>12$ years of education. Total family 12 -month income during the past year was also recorded as a continuous variable and as $\leq \$ 14,999$, $\$ 15,000$ to $\$ 24,999$, and $\geq \$ 25,000$.

For the neighborhood characteristics, census tracts were used as proxies for neighborhoods and are subdivisions of counties with an average of 4,000 residents. Consistent with previous studies, ${ }^{19,27}$ a neighborhood SES score was developed based on factor analyses of multiple 1990 U.S. Census variables. Briefly, six variables representing wealth/income (log of the median household income, log of the median value of owner occupied housing units, and the proportion of households receiving interest, dividend, or net rental income), education (the proportion of adults aged $\geq 25$ years with a high school diploma and the proportion of adults aged $\geq 25$ years with completed college education), and occupation (the proportion of employed persons aged $\geq 16$ years in executive, managerial, or professional specialty occupations) loaded into the first factor and were combined into the score. Each covariate was transformed to a $\mathrm{z}$ score by subtracting its value from the grand mean of all U.S. census tracts for that covariate and dividing the result by the standard deviation of the grand mean. The score in this sample ranged from -13.26 to 13.18 , with increasing values reflecting increasing neighborhood socioeconomic advantage. Neighborhood disadvantage as assessed using this score was previously found to be associated with poor rated oral health in blacks and whites. ${ }^{28}$ In addition to the neighborhood SES score, variables 
representing neighborhood minority composition and percentage of people living below the poverty level were investigated in the analyses.

To estimate the adjusted odds of having periodontitis, the following variables were included in the analysis: age at interview, gender, marital status, geographic region, place of birth, time since last dental visit, presence of health insurance, self-reported diabetes, and tobacco use. Age, gender, geographic region, and presence of health insurance were included in the analyses as collected in the survey. Categories in the marital status question originally included married, living together with someone as married, widowed, divorced, separated, or never married. These categories were grouped into married (married or living together with someone as married), single, divorced (separated or divorced), and widowed. Place of birth was recoded as being born in the U.S. or elsewhere. Time since last dental visit was collected as continuous in days and categorized as follows: $\leq 1$ year; $>1$ but $\geq 2$ years; $>2$ but $\leq 5$ years; and $>5$ years.

The question "Have you ever been told by a doctor that you have diabetes?" was used to assess the history of diagnosed diabetes. Diabetes reported in women only during pregnancy was excluded. Smoking status was derived from two questions: "Do you smoke cigarettes now?" and "Have you smoked at least 100 cigarettes in your entire life?" These two questions were combined to categorize respondents as current smokers (subjects who answered "Yes" to both questions), former smokers (subjects who answered "No" to the first question and "Yes" to the second question), and never smokers (subjects who answered "No" to both questions).

\section{Statistical Analysis}

Descriptive statistics for the characteristics of the population and prevalence of periodontitis were calculated by race/ethnicity, education, income, and neighborhood SES score tertiles. To determine significant differences, $\chi^{2}$ (categorical variables) and ANOVA $t$ tests (continuous variables) were used.

Logistic regression was used to estimate the strength of the association of race/ethnicity, education, income, and neighborhood SES score tertiles with the prevalence of periodontitis before and after adjusting for selected individual characteristics and risk factors. Specifically, four sets of analyses were performed to estimate the following variables: 1) crude odds ratios (OR, crude); 2) ORs adjusted for age, gender, and marital status (model 1); 3) ORs additionally mutually adjusted for race/ethnicity, education, income, and neighborhood SES score tertiles, respectively (model 2); and 4) ORs additionally adjusted for smoking and diabetes (model 3 ). These analyses were additionally adjusted for health insur- ance, dental visits, and neighborhood minority composition defined as the percentage of African Americans and Hispanics in each census tract. Interaction terms between neighborhood SES score tertiles and race/ ethnicity, income, and education were tested. These analyses were repeated using tertiles for neighborhood percentage of people living below poverty levels instead of the neighborhood SES score.

Data management procedures ${ }^{\text {29 }}$ and statistical analyses $^{\# 30}$ were carried out with the appropriate software. The statistical analysis software takes into account the complex sampling design yielding unbiased standard error estimates. In addition, because of the small number of subjects per census tract and consistent with previous studies, the statistical analysis software was used to fit marginal logistic models to account for the intraneighborhood correlation of outcomes. ${ }^{31-34}$ Marginal models account for the correlation between outcomes of individuals selected from the same neighborhoods by modeling the correlations or covariances themselves, rather than by allowing for random effects or random coefficients as multilevel models do. ${ }^{35,36}$ Thus, such correlations are taken into account in the estimation of regression coefficients and their standard errors. Therefore, the odds ratios reported are population-average rather than unit-specific estimates. In the tables, the sample sizes are unweighted. However, estimates for means, proportions, standard errors, and ORs with their $95 \%$ confidence intervals $(\mathrm{CI})$ are weighted.

\section{RESULTS}

Table 1 indicates the socioeconomic characteristics of neighborhoods in each tertile of the neighborhood SES score. Although there was enough variability in terms of race/ethnicity, education, and income in each tertile of the neighborhood SES score, blacks, Mexican Americans, and people in the lower education and income categories were more likely to live in neighborhoods classified in the lowest tertile.

The overall prevalence of periodontitis for persons aged $\geq 18$ years was $7.8 \%$ (Table 2 ), with whites exhibiting the lowest (6.8\%), blacks the highest (13.2\%), and Mexican Americans an intermediate prevalence (7.9\%; $P<0.01)$. In general, the prevalence of periodontitis was significantly higher among males, widows, those with less than a high school education, people with low income, those living in a neighborhood in the lowest tertile of the SES score, those with no dental visit in more than 5 years, those without insurance, those with diabetes, and those who currently smoked. These patterns were consistent across racial/ethnic groups, with the exception of higher prevalence of periodontitis

I SAS, SAS Institute, Cary, NC.

\# SUDAAN, Research Triangle Institute, Research Triangle Park, NC. 


\section{Table I.}

\section{Neighborhood Characteristics and Individual Measures of SES According to} Neighborhood SES Index Score: NHANES III

\begin{tabular}{|c|c|c|c|}
\hline \multirow[b]{2}{*}{ Characteristics } & \multicolumn{3}{|c|}{ Neighborhood SES Score Tertiles } \\
\hline & Lowest & Middle & Highest \\
\hline \multicolumn{4}{|l|}{ Neighborhood characteristics } \\
\hline Study participants & 4,473 & 4,185 & 4,432 \\
\hline Neighborhoods & 342 & 304 & 480 \\
\hline Neighborhood score mean $( \pm \mathrm{SD})$ & $-5.1(1.4)$ & $-1.4(0.89)$ & $3.8(2.7)$ \\
\hline Median ( \pm SD) household income $(\$)$ & | 8,478 (5,493) & $26,287(6,478)$ & $40,363(14,348)$ \\
\hline Median $( \pm S D)$ value of housing units $(\$)$ & $57,957(45,335)$ & $76,719(48,748)$ & I 47,074 $(94,644)$ \\
\hline Households with interest, dividends, or rental income (\%) & 16.0 & 32.0 & 50.0 \\
\hline Adult residents who completed high school (\%) & 47.0 & 68.0 & 86.0 \\
\hline Adult residents who completed college (\%) & 6.0 & 12.0 & 32.0 \\
\hline $\begin{array}{l}\text { Employed residents with executive, managerial, or } \\
\text { professional occupations (\%) }\end{array}$ & 12.0 & 19.0 & 35.0 \\
\hline Minority (\%) & 53.0 & 28.0 & 18.0 \\
\hline Unemployed (\%) & 14.0 & 8.0 & 5.0 \\
\hline Living below poverty (\%) & 31.0 & 16.0 & 8.0 \\
\hline \multicolumn{4}{|l|}{ Individual characteristics (n [\%]) } \\
\hline \multicolumn{4}{|l|}{ Race/ethnicity } \\
\hline Non-Hispanic whites & $619(13.8)$ & ।,792 (42.8) & $2,553(57.6)$ \\
\hline Non-Hispanic blacks & $1,905(42.6)$ & I, $185(28.3)$ & $896(20.2)$ \\
\hline Mexican Americans & I,949 (43.6) & 1,208 (28.9) & $983(22.2)$ \\
\hline \multicolumn{4}{|l|}{ Education (years) } \\
\hline$<12$ & $2,211(52.3)$ & I,497 (36.6) & $812(18.6)$ \\
\hline 12 & I,335 (31.6) & $1,526(37.3)$ & $1,406(32.2)$ \\
\hline$>12$ & $685(16.2)$ & $1,073(26.2)$ & $2,153(49.3)$ \\
\hline \multicolumn{4}{|l|}{ Income } \\
\hline$\leq \$ 14,999$ & I,879 (47.3) & I, $167(30.5)$ & $660(16.1)$ \\
\hline$\$ 15,000$ to $\$ 24,999$ & $998(25.1)$ & $941(24.6)$ & $846(20.6)$ \\
\hline$\geq \$ 25,000$ & I,094 (27.1) & ।,714 (44.8) & $2,593(63.3)$ \\
\hline
\end{tabular}

for blacks living in the Northeast and for blacks and Mexican Americans without insurance. Blacks exhibited significantly higher prevalence than whites and Mexican Americans $(P<0.01)$ across each covariate. In general, those with low education, low income, and living in neighborhoods in the lowest SES score tertile and in neighborhoods with the highest percentage of people living below poverty exhibited the highest prevalence of periodontitis across each covariate (data not shown). 
Table 2.

\section{Characteristics of the Participants in NHANES III According to Race/Ethnicity*}

\begin{tabular}{|c|c|c|c|c|c|c|c|c|}
\hline \multirow[b]{2}{*}{ Characteristics } & \multicolumn{2}{|c|}{$\begin{array}{l}\text { African Americans } \\
\qquad(\mathrm{n}=3,986)\end{array}$} & \multicolumn{2}{|c|}{$\begin{array}{l}\text { Mexican Americans } \\
\qquad(n=4,140)\end{array}$} & \multicolumn{2}{|c|}{$\begin{array}{l}\text { Non-Hispanic Whites } \\
\qquad(n=4,964)\end{array}$} & \multicolumn{2}{|c|}{ Total $^{\dagger}(n=13,665)$} \\
\hline & $\%^{\ddagger}$ & Periodontitis (\%) & $\%$ & Periodontitis (\%) & $\%$ & Periodontitis (\%) & $\%$ & Periodontitis (\%) \\
\hline Age (years) ${ }^{\S}$ & $37.4(0.37)$ & 13.2 & $35.1(0.44)$ & 7.9 & $41.2(0.50)$ & 6.8 & $40.1(0.37)$ & 7.8 \\
\hline \multicolumn{9}{|l|}{ Gender } \\
\hline Male & $44.6(1.06)$ & 17.6 & $52.3(0.72)$ & 10.4 & $49.1(0.53)$ & 8.6 & $48.7(0.48)$ & 9.9 \\
\hline Female & $55.4(1.06)$ & 9.7 & $47.7(0.72)$ & 5.2 & $50.9(0.53)$ & 5.0 & $51.3(0.48)$ & 5.7 \\
\hline \multicolumn{9}{|l|}{ Marital status } \\
\hline Married & $41.2(1.04)$ & 15.1 & $65.0(1.53)$ & 9.2 & $68.1(1.11)$ & 7.1 & $63.6(0.94)$ & 7.9 \\
\hline Divorced & | $8.7(0.79)$ & 15.6 & | $1.0(0.68)$ & 9.0 & $10.1(0.56)$ & 9.6 & $11.5(0.44)$ & 11.3 \\
\hline Single & $35.1(1.12)$ & 7.4 & $21.7(1.29)$ & 2.6 & $17.7(1.08)$ & 2.1 & $20.9(0.87)$ & 3.2 \\
\hline Widow & $4.9(0.37)$ & 28.6 & $2.3(0.31)$ & 19.7 & $4.1(0.32)$ & 13.6 & $4.0(0.25)$ & 17.4 \\
\hline \multicolumn{9}{|l|}{ Geographic region } \\
\hline Northeast & I5.8(1.66) & 14.2 & $1.3(0.34)$ & 3.9 & $21.4(1.60)$ & 7.3 & $19.9(1.16)$ & 8.1 \\
\hline Midwest & $19.8(1.58)$ & 12.5 & $10.7(2.90)$ & 7.8 & $26.6(1.73)$ & 7.1 & $23.5(1.31)$ & 7.7 \\
\hline South & $55.3(2.52)$ & 13.1 & $27.1(5.30)$ & 10.7 & $32.7(3.01)$ & 7.4 & $34.6(2.69)$ & 8.6 \\
\hline West & $9.1(1.42)$ & 13.8 & $60.8(6.17)$ & 6.8 & $19.3(4.14)$ & $4.6 *$ & $22.0(3.62)$ & 6.1 \\
\hline \multicolumn{9}{|l|}{ Nativity status } \\
\hline U.S. born & & & $44.8(3.26)$ & 8.3 & & & $96.2(0.49)$ & 7.2 \\
\hline Foreign & & & $55.2(3.26)$ & $7.6 *$ & & & $3.8(0.49)$ & 7.5 \\
\hline \multicolumn{9}{|l|}{ Education (years) } \\
\hline$<12$ & $28.2(1.37)$ & 18.8 & $55.8(1.69)$ & 9.9 & $15.8(0.96)$ & 13.7 & 21.1 (0.88) & 13.2 \\
\hline 12 & $40.3(1.20)$ & 13.1 & $26.4(0.98)$ & 5.6 & $35.7(1.07)$ & 7.1 & $34.9(0.82)$ & 8.2 \\
\hline$>12$ & $31.6(1.46)$ & 7.9 & $17.8(1.22)$ & 2.3 & $48.5(1.57)$ & 4.2 & $44.0(1.28)$ & 4.6 \\
\hline \multicolumn{9}{|l|}{ Income } \\
\hline$\leq \$ 14,999$ & $37.5(2.03)$ & I5.1 & $36.6(1.50)$ & 10.2 & I 3.9 (0.79) & 10.4 & $19.0(0.99)$ & 11.3 \\
\hline$\$ 15,000$ to $\$ 24,999$ & $22.6(1.32)$ & 12.7 & $27.4(1.12)$ & 8.2 & $18.4(1.08)$ & 8.4 & $20.0(0.86)$ & 9.2 \\
\hline$\geq \$ 25,000$ & $39.9(1.90)$ & 11.6 & $35.9(1.58)$ & 5.5 & $67.6(1.38)$ & 5.4 & $61.0(1.26)$ & 6.0 \\
\hline \multicolumn{9}{|c|}{ Neighborhood score tertiles } \\
\hline High & $44.7(3.67)$ & 15.3 & $40.4(3.73)$ & 10.2 & $10.2(1.32)$ & 10.8 & $17.1(1.42)$ & 12.1 \\
\hline Medium & $29.3(3.5 I)$ & 13.1 & $31.3(3.01)$ & 8.1 & $34.3(2.49)$ & 8.8 & $33.2(2.08)$ & 9.2 \\
\hline Low & $25.9(2.74)$ & 9.8 & $28.2(2.80)$ & 4.6 & $55.5(2.84)$ & 4.9 & $49.7(2.45)$ & 5.4 \\
\hline \multicolumn{9}{|l|}{ Poverty tertiles } \\
\hline High & $47.2(3.01)$ & 15.1 & $36.9(3.82)$ & 10.8 & $8.3(0.92)$ & 10.8 & $16.0(1.14)$ & 11.9 \\
\hline Medium & $27.3(1.98)$ & 14.2 & $35.6(2.45)$ & 6.8 & $34.1(2.62)$ & 9.0 & $33.4(2.08)$ & 9.4 \\
\hline Low & $25.4(2.68)$ & 9.0 & $27.4(2.72)$ & 5.8 & $57.6(2.61)$ & 4.9 & $50.6(2.26)$ & 5.5 \\
\hline \multicolumn{9}{|c|}{ Minority composition tertiles } \\
\hline Low & $70.1(2.05)$ & 14.4 & $43.1(3.92)$ & 8.2 & $8.3(2.05)$ & 9.0 & $20.4(1.28)$ & 10.7 \\
\hline Medium & $21.5(1.95)$ & 10.4 & $41.8(3.11)$ & 8.5 & $22.6(2.06)$ & 5.8 & $25.0(1.91)$ & 7.1 \\
\hline High & $8.4(0.97)$ & 11.2 & $15.2(2.08)$ & 5.7 & $69.2(2.18)$ & 6.8 & $54.6(2.15)$ & 7.0 \\
\hline \multicolumn{9}{|c|}{ Time since last dental visit } \\
\hline$\leq 1$ year & $58.6(1.23)$ & 10.5 & $55.3(1.47)$ & 6.7 & $72.3(1.19)$ & 5.9 & $69.6(0.93)$ & 6.6 \\
\hline$>1$ but $\leq 2$ years & $14.5(0.66)$ & 14.2 & | $4.7(0.66)$ & 7.9 & $12.7(0.73)$ & 6.6 & $12.9(0.58)$ & 7.7 \\
\hline$>2$ but $\leq 5$ years & $14.6(0.90)$ & 17.2 & $17.0(0.65)$ & 8.3 & $9.1(0.61)$ & 10.3 & $\mid 0.5(0.5 \mid)$ & 10.9 \\
\hline$>5$ years & $12.3(0.67)$ & 21.1 & | $3.1(0.53)$ & 10.5 & $5.9(0.39)$ & 12.2 & $7.0(0.33)$ & 13.8 \\
\hline
\end{tabular}


Table 2. (continued)

\section{Characteristics of the Participants in NHANES III According to Race/Ethnicity*}

\begin{tabular}{|c|c|c|c|c|c|c|c|c|}
\hline \multirow[b]{2}{*}{ Characteristics } & \multicolumn{2}{|c|}{$\begin{array}{l}\text { African Americans } \\
\qquad(\mathrm{n}=3,986)\end{array}$} & \multicolumn{2}{|c|}{$\begin{array}{l}\text { Mexican Americans } \\
\qquad(n=4,140)\end{array}$} & \multicolumn{2}{|c|}{$\begin{array}{l}\text { Non-Hispanic Whites } \\
\qquad(n=4,964)\end{array}$} & \multicolumn{2}{|c|}{ Total $^{\dagger}(n=13,665)$} \\
\hline & $\%^{\ddagger}$ & Periodontitis (\%) & $\%$ & Periodontitis (\%) & $\%$ & Periodontitis (\%) & $\%$ & Periodontitis (\%) \\
\hline \multicolumn{9}{|c|}{ Presence of health insurance } \\
\hline Yes & $82.8(1.21)$ & 12.1 & $88.0(0.93)$ & 7.4 & $94.4(0.46)$ & 6.3 & $93.2(0.39)$ & 7.0 \\
\hline No & $17.2(1.21)$ & 10.7 & $12.0(0.93)$ & 6.4 & $5.6(0.46)$ & $8.4 *$ & $6.8(0.39)$ & 8.3 \\
\hline \multicolumn{9}{|l|}{ Diabetes } \\
\hline Yes & $4.6(0.37)$ & 30.7 & $4.4(0.29)$ & 28.1 & $3.5(0.33)$ & 15.6 & $3.6(0.24)$ & 17.8 \\
\hline No & $95.4(0.33)$ & 12.4 & $95.6(0.29)$ & 7.0 & $96.5(0.33)$ & 6.5 & $96.4(0.24)$ & 7.4 \\
\hline \multicolumn{9}{|l|}{ Smoking status } \\
\hline Current & $32.4(1.03)$ & 19.1 & $22.4(0.91)$ & 11.5 & $28.9(1.12)$ & 11.3 & $28.2(0.87)$ & 12.9 \\
\hline Former & |4.| (0.69) & 16.9 & $18.8(0.72)$ & 13.2 & $25.7(0.65)$ & 8.4 & $23.0(0.62)$ & 9.1 \\
\hline Never & $53.6(1.16)$ & 8.7 & $58.9(0.82)$ & 4.9 & $45.3(0.95)$ & 3.0 & $48.8(0.80)$ & 4.1 \\
\hline
\end{tabular}

* All ns are unweighted, whereas means, proportions, and standard errors are weighted to take into account NHANES III's sample design; $P>0.05$.

† Total includes "others."

\# Proportions ( \pm SE).

$\S$ Means $( \pm \mathrm{SE})$.

Table 3 presents the crude and adjusted ORs and their $95 \% \mathrm{CI}$ for periodontitis for race/ethnicity, education, income, and neighborhood SES score tertiles. Periodontitis was associated with race/ethnicity, education, income, and neighborhood SES score. Blacks were more than twice as likely to have periodontitis as whites (OR: 2.10; 95\% Cl: 1.70, 2.59). After adjusting for age, gender, marital status, education, income, and neighborhood SES score, this association remained similar to the crude association (OR: 1.99; $95 \%$ CI: $1.58,2.53)$. There was no statistically significant difference in the odds of having periodontitis for Mexican Americans versus whites.

Compared to those with more than a high school education, those with less than a high school diploma were three times (95\% CI: $2.40,4.06)$ more likely to have periodontitis. When age, gender, marital status, race/ethnicity, income, and neighborhood SES score were accounted for, this association decreased to 2.07 (95\% CI: 1.48, 2.89). Further adjustment for diabetes and smoking substantially decreases the strength of the association between education and periodontitis. However, the association remains significant (OR: $1.67 ; 95 \%$ CI: $1.19,2.34)$. A significant trend for this association was observed through all the models.

Low income and living in neighborhoods in the lowest tertile of the SES score were significantly associated with higher odds of having periodontitis (OR: 2.00; 95\% CI: 1.62, 2.47 and OR: 2.41; 95\% CI: $1.88,3.08$, respectively). However, after adjusting for age, gender, marital status, race/ethnicity, education, and income, the association remains significant for neighborhood SES score only (OR: $1.81 ; 95 \% \mathrm{CI}$ :
1.36, 2.41). After additional adjustment for diabetes and smoking, the association between neighborhood SES score and periodontitis remains significant (OR: 1.73; 95\% CI: 1.29, 2.32). The finding remains essentially unchanged when additional analysis was performed adjusting for health insurance and dental visits (data not shown). Finally, we repeated this analysis additionally adjusting for the proportion of minority populations in the neighborhood, and the result remained essentially the same (data not shown). There was a significant trend for the association between neighborhood SES score and periodontitis.

There was no interaction between neighborhood score tertiles and race/ethnicity, income, or education. When tertiles for neighborhood percentage of people living below poverty were used instead of the neighborhood SES score, the results were similar to those presented using the neighborhood SES score in model 3 (OR for higher tertile of percentage of people living below poverty: 1.69; 95\% CI: 1.26, 2.27; data not shown in Table 3). These results remained the same when the analysis was repeated with additional adjustment for neighborhood minority composition.

All analyses were repeated after adjusting for the mean number of teeth and for region of the country, and the results for the associations of race/ethnicity, income, education, and neighborhood SES score with periodontitis remained nearly unchanged (data not shown).

\section{DISCUSSION}

Race/ethnicity, education, and neighborhood SES conditions were associated with increased odds of 
Table 3.
ORs* (95\% CIs) for Periodontitis for Race/Ethnicity, Personal Income, Education, and Neighborhood SES Tertiles

\begin{tabular}{|c|c|c|c|c|}
\hline \multirow[b]{2}{*}{ Characteristics } & \multicolumn{4}{|c|}{ Periodontitis } \\
\hline & Crude & Model I & Model 2 & Model 3 \\
\hline \multicolumn{5}{|l|}{ Race/ethnicity } \\
\hline Non-Hispanic whites & 1.00 & 1.00 & 1.00 & 1.00 \\
\hline Non-Hispanic blacks & $2.10(1.70,2.59)$ & $2.86(2.28,3.60)$ & $1.99(1.58,2.53)$ & $2.01(1.69,2.55)$ \\
\hline Mexican Americans & $1.19(0.92,1.52)$ & $1.74(1.32,2.27)$ & $1.05(0.78,1.40)$ & $1.33(1.00,1.77)$ \\
\hline \multicolumn{5}{|l|}{ Education (years) } \\
\hline$<12$ & $3.12(2.40,4.06)$ & $2.76(2.15,3.56)$ & $2.07(1.48,2.89)$ & $1.67(1.19,2.34)$ \\
\hline 12 & $1.84(1.47,2.31)$ & $2.00(1.59,2.52)$ & $1.68(1.31,2.15)$ & $1.45(1.11,1.89)$ \\
\hline$>12$ & 1.00 & 1.00 & 1.00 & 1.00 \\
\hline$P$ trend & $<0.000$ । & $<0.000$ । & 0.0001 & 0.01 \\
\hline \multicolumn{5}{|l|}{ Income } \\
\hline$\leq \$ 14,999$ & $2.00(1.62,2.47)$ & $2.11(1.71,2.61)$ & $1.29(0.96,1.74)$ & $1.24(0.91,1.67)$ \\
\hline$\$ 15,000$ to $\$ 24,999$ & $1.59(1.23,2.05)$ & $1.58(1.20,2.09)$ & $1.16(0.85,1.58)$ & $1.13(0.82,1.55)$ \\
\hline$\geq \$ 25,000$ & 1.00 & 1.00 & 1.00 & 1.00 \\
\hline$P$ trend & $<0.000$ । & $<0.000$ । & 0.21 & 0.35 \\
\hline \multicolumn{5}{|c|}{ Neighborhood score tertiles } \\
\hline Low & $2.41(1.88,3.08)$ & $2.94(1.72,2.59)$ & $1.81(1.36,2.4 I)$ & $1.73(1.29,2.32)$ \\
\hline Medium & $1.78(1.36,2.32)$ & $1.96(1.50,2.55)$ & $1.63(1.23,2.16)$ & $1.63(1.23,2.17)$ \\
\hline High & 1.00 & 1.00 & 1.00 & 1.00 \\
\hline$P$ trend & $<0.0001$ & $<0.000 \mid$ & $<0.0005$ & 0.003 \\
\hline
\end{tabular}

* Model 1 adjusted for age and gender; model 2 additionally adjusted for marital status, race/ethnicity, education, income, and neighborhood score and vice versa; and model 3 additionally adjusted for traditional risk factors (smoking and diabetes).

having periodontitis in NHANES III. Specifically, blacks, those with low education, and those living in neighborhoods in the lowest tertile of the SES score were more likely to have periodontitis. These results remained significant after adjusting for age, gender, race/ethnicity, marital status, diabetes, smoking, education, income, and neighborhood SES score.

Previous studies have documented differences in periodontal diseases by race/ethnicity and socioeconomic indicators, with most studies reporting higher prevalence of periodontal diseases for blacks and those with low income and less education. ${ }^{2-4,7,10,17,22}$ However, these studies have included individual-level characteristics only with one exception. A study using data from the Atherosclerosis Risk in Communities (ARIC) ${ }^{37}$ study and race-specific analysis found low income and low education to be associated with severe periodontitis in African Americans, whereas only low income was associated with severe periodontitis in whites after accounting for neighborhood SES conditions. It is worth noting that socioeconomic conditions in neighborhoods in which African Americans reside, defined using the same score as in this study, were worse than the ones in which whites live. Therefore, individual resources, such as income and education, may be more important for periodontal diseases among African Americans. Contrary to previous studies, our study did not find an association between individual income and periodontitis after additionally accounting for neighborhood SES circumstances. However, our study, consistent with previous studies, showed an association between race/ethnicity and education with periodontitis, with blacks and those with less education exhibiting higher odds of having periodontitis than their white or Mexican American and more-educated counterparts. These associations persist after adjusting for neighborhood SES conditions and other relevant individual characteristics. This finding suggests that individual income may not be as important for periodontal diseases under equal neighborhood SES conditions. However, the effects of race and education are pervasive independent of the neighborhood SES circumstances. Race as a social construct may represent a mix of exposures independent of individual income and neighborhood SES conditions that may act directly or indirectly upon periodontal diseases. ${ }^{38}$ Education, on the other hand, seems to have an effect on health independent of other 
economic resources. ${ }^{39,40}$ The ARIC study presented race-specific analyses to account for the fact that almost the entire African American sample was selected from a single locale (Jackson, Mississippi), whereas our study treated race/ethnicity as an independent variable during the analyses. This difference could explain the dissimilarity in findings between studies. Finally, African Americans in the ARIC study were recruited predominantly from a single geographic locale, precluding the ability to make inferences to African Americans in other communities or at the national level.

To our knowledge, this is the first national-level study published to investigate neighborhood effects on periodontal diseases. In general, previous studies suggest that an area of residence may influence an individual's health behaviors and health-related norms. ${ }^{19,21}$ For periodontal diseases in particular, social contexts could promote or prevent behaviors that affect periodontal health, such as oral health habits, eating patterns, and cigarette smoking. This study found that individuals living in the lowest tertile of the neighborhood SES score or in more disadvantaged neighborhoods were almost twice as likely to have periodontitis as their counterparts living in neighborhoods in the highest tertile of the SES score after accounting for their race/ethnicity, income, education, and other relevant characteristics.

Consistent with previous studies examining neighborhood effects on health and mortality in the U.S., our study used census tracts (mean population: 4,000 ) to define neighborhoods. In addition, we used aggregate measures from the U.S. Census Bureau to determine the neighborhood SES characteristics, and further, define the score. Although these measures may represent summaries of individual characteristics, making it difficult to separate neighborhood SES effects from the effects of individual-level SES indicators, ${ }^{41}$ we did not find strong correlations between neighborhood SES score and individual income and education (Spearman $r=0.32$ and 0.34 , respectively). This finding could suggest that individual income and education may be tapping into constructs not captured by neighborhood SES conditions. Furthermore, although this study found appreciable variability in study participants' race/ethnicity, income, and education distributions across neighborhood SES score tertiles (Table 1), we did not find that the associations of individual race/ethnicity, income, or education with periodontitis vary by neighborhood SES score tertiles.

Among the strengths of our study are the use of nationally representative data and the large sample size, which allow the control of several potential confounders and the examination of interactions. Important shortcomings are the limitations of all observational studies in their ability to tease out compositional (i.e., individual) and contextual (i.e., neighborhood) effects. Although standard multivariable approaches were used to control the association between neighborhood-level SES and periodontal diseases for individual-level socioeconomic indicators and vice versa, it is possible that these variables may mediate association rather than confound it. ${ }^{19,21}$ Moreover, the cross-sectional nature of the data and the lack of information on the length of time individuals have been disadvantaged or the length of time individuals have been residing in their neighborhoods prevent us from making inferences regarding temporality between exposure and disease. Finally, a limitation inherent in national surveys collecting periodontal data is the use of a partial-mouth recording examining only two sites (mesio-buccal and midfacial) in two randomly selected quadrants, under the assumption that these measurements are representative of the full mouth. ${ }^{42,43}$ Therefore, the estimates presented here could have underestimated the prevalence of periodontitis.

This study indicated that race/ethnicity, individual education, and neighborhood SES circumstances are associated with periodontal health. However, to determine whether neighborhood SES is causally related to population levels of periodontal diseases, studies designed to examine the specific processes through which neighborhood characteristics act upon or interact with individual-level SES indicators and the behaviors that affect periodontal diseases will have to be conducted. Specifically, it is imperative to determine how the social milieu and individual characteristics interact to shape individual health behaviors to prevent or exacerbate periodontal diseases. This type of research could help promote our goal of eliminating racial/ethnic and economic oral health disparities.

\section{ACKNOWLEDGMENTS}

This work was supported by a grant from the National Institute of Dental and Craniofacial Research, Bethesda, Maryland (K22DE15317 to LNB) and the Robert Wood Johnson Foundation Health \& Society Scholars Program, Princeton, New Jersey (to LNB).

\section{REFERENCES}

1. Albandar JM. Periodontal diseases in North America. Periodontol 2000 2002;29:31-69.

2. Douglass CW, Gillings D, Sollecito W, Gammon M. National trends in the prevalence and severity of the periodontal diseases. J Am Dent Assoc 1983;107: 403-412.

3. Borrell LN, Burt BA, Gillespie BW, Lynch JW, Neighbors $\mathrm{H}$. Race and periodontitis in the US: Beyond black and white. J Public Health Dent 2002;62:92-101.

4. Nikias MK, Fink R, Sollecito W. Oral health status in relation to socioeconomic and ethnic characteristics 
of urban adults in the U.S.A. Community Dent Oral Epidemiol 1977;5:200-206.

5. Oliver RC, Brown LJ, Löe H. Variations in the prevalence and extent of periodontitis. J Am Dent Assoc 1991;122:43-48.

6. Oliver RC, Brown LJ, Löe H. Periodontal diseases in the United States population. J Periodontol 1998;69: 269-278.

7. Elter JR, Beck JD, Slade GD, Offenbacher S. Etiologic models for incident periodontal attachment loss in older adults. J Clin Periodontol 1999;26: 113-123.

8. Borrell LN, Lynch JW, Neighbors H, Burt BA, Gillespie $\mathrm{BW}$. Is there homogeneity in periodontal health between African Americans and Mexican Americans? Ethn Dis 2002;12:97-110.

9. Locker D, Leake JL. Risk indicators and risk markers for periodontal disease experience in older adults living independently in Ontario, Canada. J Dent Res 1993;72:9-17.

10. U.S. Department of Health and Human Services. Oral Health in America: A Report of the Surgeon General. Rockville, MD: U.S. Department of Health and Human Services, National Institute of Dental and Craniofacial Research, National Institutes of Health; 2000.

11. White BA, Caplan DJ, Weintraub JA. A quarter century of changes in oral health in the United States. $J$ Dent Educ 1995;59:19-57.

12. Caplan DJ, Weintraub JA. The oral health burden in the United States: A summary of recent epidemiologic studies. J Dent Educ 1993;57:853-862.

13. Brown LJ, Brunelle JA, Kingman A. Periodontal status in the United States, 1988-1991: Prevalence, extent, and demographic variation. J Dent Res 1996;75: 672-683.

14. Kelly JE, Van Kirk LE. Periodontal disease in adults. Vital Health Stat 11 1965;(12):1-30.

15. Oral Health Coordinating Committee, Public Health Service. Toward improving the oral health of Americans: An overview of oral health status, resources, and care delivery. Public Health Rep 1993;108: 657-672.

16. Albandar JM, Brunelle JA, Kingman A. Destructive periodontal disease in adults 30 years of age and older in the United States, 1988-1994. J Periodontol 1999; 70:13-29.

17. Borrell LN, Burt BA, Neighbors HW, Taylor GW. Social factors and periodontitis in an older population. Am J Public Health 2004;94:748-754.

18. Pickett KE, Pearl M. Multilevel analyses of neighbourhood socioeconomic context and health outcomes: A critical review. J Epidemiol Community Health 2001; 55:111-122.

19. Diez Roux AV, Merkin SS, Arnett D, et al. Neighborhood of residence and incidence of coronary heart disease. N Engl J Med 2001;345:99-106.

20. Krieger N, Chen JT, Waterman PD, Soobader M-J, Subramanian SV, Carson R. Geocoding and monitoring of US socioeconomic inequalities in mortality and cancer incidence: Does the choice of area-based measure and geographic level matter? The Public Health Disparities Geocoding Project. Am J Epidemiol 2002; 156:471-482.

21. Macintyre S, Ellaway A, Cummins S. Place effects on health: How can we conceptualise, operationalise and measure them? Soc Sci Med 2002;55:125-139.
22. Burt BA, Eklund SA. Dentistry, Dental Practice and the Community, 6th ed. St. Louis: Elsevier Saunders, 2005.

23. Andersen RM, Davidson PL. Ethnicity, aging, and oral health outcomes: A conceptual framework. Adv Dent Res 1997;11:203-209.

24. National Center for Health Statistics. Plan and operation of the Third Health and Nutrition Examination Survey, 1988-94. Vital Health Stat 1 1994;(32):1-412.

25. Machtei EE, Christersson LA, Grossi SG, Dunford R, Zambon JJ, Genco RJ. Clinical criteria for the definition of "established periodontitis." J Periodontol 1992; 63:206-214.

26. Beck JD, Koch GG, Rozier RG, Tudor GE. Prevalence and risk indicators for periodontal attachment loss in a population of older community-dwelling blacks and whites. J Periodontol 1990;61:521-528.

27. Diez-Roux AV, Kiefe CI, Jacobs DR Jr., et al. Area characteristics and individual-level socioeconomic position indicators in three population-based epidemiologic studies. Ann Epidemiol 2001;11:395-405.

28. Borrell LN, Taylor GW, Borgnakke WS, Woolfolk MW, Nyquist LV. Perception of general and oral health in White and African American adults: Assessing the effect of neighborhood socioeconomic conditions. Community Dent Oral Epidemiol 2004;32: 363-373.

29. SAS Institute Inc. SAS/STAT 9.1 User's Guide. Cary, NC: SAS Institute; 2004.

30. Research Triangle Institute. SUDAAN Language Manual, Release 9.0. Research Triangle Park, NC: Research Triangle Institute; 2004.

31. Cubbin C, Hadden WC, Winkleby MA. Neighborhood context and cardiovascular disease risk factors: The contribution of material deprivation. Ethn Dis 2001; 11:687-700.

32. Diez Roux A, Merkin SS, Arnet D, et al. Neighborhood of residence and incidence of coronary heart disease. N Engl J Med 2001;345:99-106.

33. LeClere FB, Rogers RG, Peters KD. Ethnicity and mortality in the United States: Individual and community correlates. Soc Forces 1997;76:169-198.

34. Winkleby MA, Cubbin C. Influence of individual and neighbourhood socioeconomic status on mortality among black, Mexican-American, and white women and men in the United States. J Epidemiol Community Health 2003;57:444-452.

35. Zeger SL, Liang KY, Albert PS. Models for longitudinal data: A generalized estimating equation approach. Biometrics 1988;44:1049-1060.

36. Diggle PJ, Heagerty P, Liang KY, Zeger SL. Analysis of Longitudinal Data, 2nd ed. New York: Oxford University Press; 2002:126-128, 141-160.

37. Borrell LN, Beck JD, Heiss G. Socioeconomic disadvantage and periodontal disease: The Dental Atherosclerosis Risk in Communities study. Am J Public Health 2006;96:332-339.

38. Williams DR. Race, socioeconomic status, and health. The added effects of racism and discrimination. Ann $N$ $Y$ Acad Sci 1999;896:173-188.

39. Lynch J, Kaplan G. Socioeconomic position. In: Berkman LF, Kawachi I, eds. Social Epidemiology. New York, NY: Oxford University Press; 2000:13-35.

40. Krieger $N$, Williams DR, Moss NE. Measuring social class in US public health research: Concepts, methodologies, and guidelines. Annu Rev Public Health 1997;18:341-378. 
41. Oakes JM. The (mis)estimation of neighborhood effects: Causal inference for a practicable social epidemiology. Soc Sci Med 2004;58:1929-1952.

42. Kingman A, Albandar JM. Methodological aspects of epidemiological studies of periodontal diseases. Periodontol 2000 2002;29:11-30.

43. Albandar JM, Brunelle JA, Kingman A. Destructive periodontal disease in adults 30 years of age and older in the United States, 1988-1994. J Periodontol 1999; 70:13-29.

Correspondence: Dr. Luisa N. Borrell, Department of Epidemiology, Mailman School of Public Health, Columbia University, 722 W. $168^{\text {th }}$ St., New York, NY 10032. Fax: 212/544-4221; e-mail: Inb2@columbia.edu.

Accepted for publication September 9, 2005. 